\title{
On the role of initial void geometry in plastic deformation of metallic thin films: A molecular dynamics study
}

\author{
Yanqing $\mathrm{Su}^{\mathrm{a}}$, Shuozhi $\mathrm{Xu}^{\mathrm{b}, *}$ \\ ${ }^{a}$ School of Earth and Atmospheric Sciences, Georgia Institute of Technology, Atlanta, \\ GA 30332-0340, USA \\ ${ }^{b} G W W$ School of Mechanical Engineering, Georgia Institute of Technology, Atlanta, GA \\ 30332-0405, USA
}

\begin{abstract}
Void growth is usually considered one of the most critical phases leading to dynamic fracture of ductile materials. Investigating the detailed process of void growth at the nanoscale aids in understanding the damage mechanism of metals. While most atomistic simulations by far assume circular or spherical voids for simplicity, recent studies highlight the significance of the initial void ellipticity in mechanical response of voided metals. In this work, we perform large scale molecular dynamics simulations with millions of atoms to investigate the void growth in plastic deformation of thin films in face-centered cubic $\mathrm{Cu}$. It is found that the initial ellipticity and the initial orientation angle of the void have substantial impacts on the dislocation nucleation, the void evolution, and the stress-strain response. In particular, the initial dislocation emission sites and the sequence of slip plane activation vary with the initial void geometry. For the void size evolution, three regimes are identified:
\end{abstract}

\footnotetext{
${ }^{*}$ Corresponding author

Email address: shuozhixu@gatech.edu (Shuozhi Xu)
} 
(I) the porosity increases relatively slowly in the absence of dislocations, (II) the porosity grows much rapidly after dislocations start to glide on different slip planes, and (III) the rate of porosity variation becomes much more slowly when dislocations are saturated in the model, and the void surface becomes irregular, non-smooth. In terms of the stress-strain response, the effects of the initial orientation angle are more pronounced when the initial void ellipticity is large; the influence of the initial void ellipticity is different for different initial orientation angles. The effects of the temperature, the strain rate, the loading direction, and the initial porosity in the void growth are also explored. Our results reveal the underlying mechanisms of initial void geometry-dependent plastic deformation of metallic thin films and shed light on informing more accurate theoretical models.

Keywords: Molecular dynamics, metals, nanovoid, ellipticity, plastic deformation

\section{Introduction}

Voids, frequently introduced during manufacturing, processing, or irradiation, play a significant role in mechanical properties of metals by altering the microstructure and influencing dislocation motion [1-3]. Dislocationvoid interactions are important mechanisms of the strain hardening in which a higher applied shear stress is required for dislocations to bypass even nmsized voids [4]. When ductile materials are subject to a tensile loading, voids usually start to form at the interface between inclusions and matrix, before growing and coalescing each other, facilitating the damage and fracture [5]. The growth and coalescence of voids have a great impact on the 
local structure of grain boundaries $(\mathrm{GBs})$ in nanocrystals [6], as well as on interstitial loops and point defects in irradiated materials, promoting their damage tolerance $[7,8]$. Thus, exploring the dynamics of void growth can help understanding the plastic deformation of metallic materials with different microstructures in various types of environments.

In the last decade, the finite element method (FEM) has been employed to study void growth and coalescence as a function of many variables, including the temperature, void configuration [9], stress triaxiality [10], crystallographic orientations [11], initial void volume fraction [12], initial void size [13], and initial void shape [14]. However, as the size of void approaches nanoscale, the continuum approximation based on which FEM is established is not valid anymore. For example, it is challenging for FEM to capture the dislocation emission and the phase transformation during the void growth and coalescence, the latter of which is usually considered one of the most critical stages leading to the dynamic ductile fracture [15]. For phenomena occurring at the nanoscale, atomistic simulations such as molecular dynamics (MD) are more suitable to study the lattice defect evolution involved in the process of void growth.

In recent years, numerous researchers have employed MD simulations to investigate the issue of nanovoid growth. Traiviratana et al. [16] found that the emission of dislocation loops is the primary mechanism of void growth in face-centered cubic (FCC) $\mathrm{Cu}$ when the specimen is subject to tensile uniaxial strains; Potirniche et al. [17] revealed a pronounced effect of the specimen size on dislocation formation from voids and plastic flow in FCC Ni; Chang et al. [18] showed that with an increasing temperature, the yield 
stress decreases while the void growth rate remains invariant; Bringa et al. [19] studied the effect of the loading orientation in the void behavior in $\mathrm{Cu}$, and their results exhibited a complex coupling of void growth, GB debonding, and partial dislocation emission into grains in nanocrystals; Seppälä et al. [20] quantified the effect of stress triaxiality on the void growth in $\mathrm{Cu}$; Deng et al. [15] uncovered a strong configurational effect on the coalescence of voids in $\mathrm{Cu}$ under a shock loading. Using the quasicontinuum method, Marian et al. [21] and Ponga et al. [22] studied the nanovoid deformation in FCC Al under tension and simple shear, identifying a transitional strain rate between a quasistatic and a dynamic regime. The void growth in other types of lattice, including the body-centered cubic (BCC) [23] and hexagonal closepacked (HCP) systems [5], has also been probed. In these systems, it is the twinning and phase transformation, other than the dislocation nucleatoin, that dominate the plasticity [24].

In establishing the initial configuration for the void growth problem, one of the most important geometric factors - among specimen size, porosity, and lattice orientations - is the void shape. For simplicity, most theoretical and computational work assume circular or spherical voids [25]. Recent studies found that, however, an initially circular void can deform to an elliptic shape of the same volume subject to either radiation damage or dislocation glide [26]. In 3-D, an initially spherical void could develop facets as a result of the prismatic and shear loops formation at its surface, accounting for the generation of geometrically necessary dislocations required for void growth [27]. These findings suggest that non-circular and non-spheric voids are prevalent in real materials. Therefore, it will be a more generalized treat- 
ment of the void shape to employ elliptic voids in initial simulation setups. On the other hand, while theoretical models based on continuum elastic theory have been developped to take into account the effects of ellipticity on the void growth in plastic deformation of metals [28], mechanisms for such effects at the nanoscale remain largely unknown. Recently, an attempt has been made to investigate the growth and coalescence of initially elliptic voids in Al, which highlights the significance of the initial void ellipticity in mechanical responses of voided metallic thin films subject to tension $[29,30]$. Nevertheless, to our best knowledge, a systematic study of the effects of the geometry of an initially elliptic void on plastic deformation of metals is still lacking.

In this work, large scale MD simulations with millions of atoms are performed to systematically study the effects of the initial void ellipticity and the initial void orientation angle in plastic deformation of metallic thin films in FCC $\mathrm{Cu}$, which has a large elastic anisotropy index of about 3.22. Emphasis will be placed on how these two factors influence the dislocation emission, the void evolution, and the stress-strain response.

\section{Material and methods}

The simulation cell containing a single nanovoid in a thin film is shown in Fig. 1. Periodic boundary conditions (PBCs) are applied along all directions to simulate a periodic array of voids in an infinite system. A cylindrical void, whose cross-sectional area on the $y$ - $z$ plane has an ellipticity $e=\sqrt{\left(a^{2}-c^{2}\right) / a^{2}}$, is positioned in the center of the cell, where $a$ and $c$ are the length of the major and minor axes, respectively. The centroid of the void 


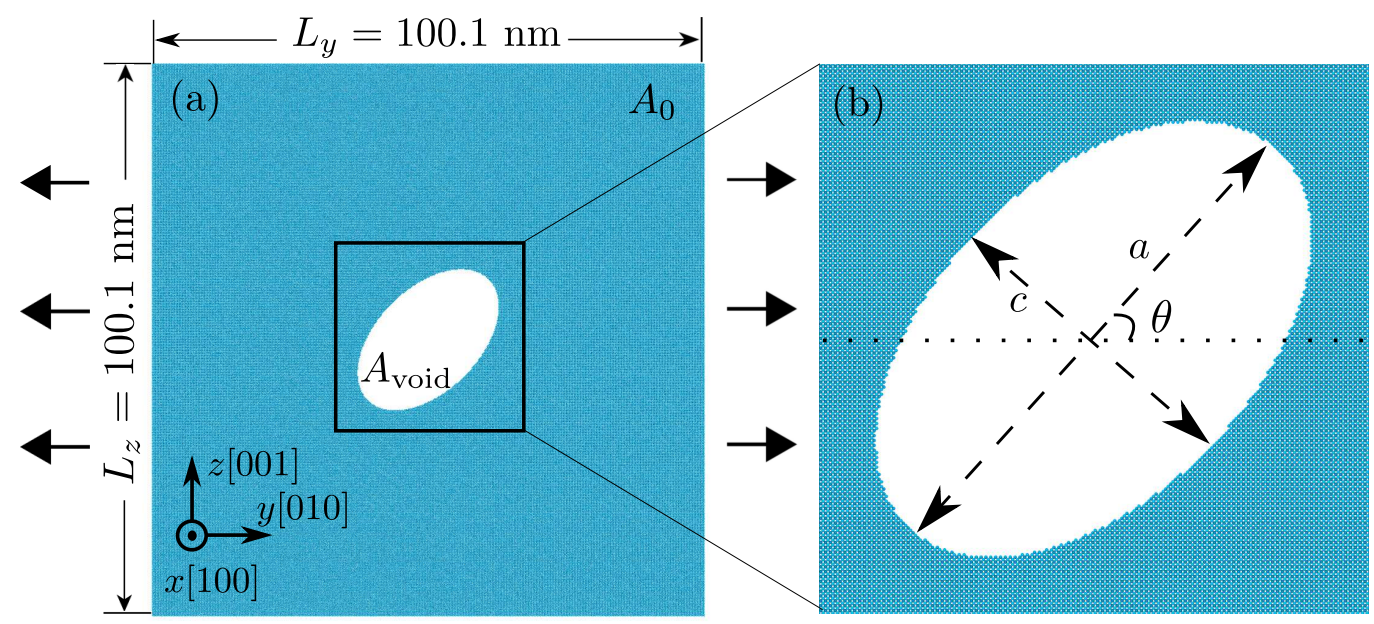

Figure 1: Simulation cell of a metallic thin film containing a void. Depth of the model in one supercell $L_{x}=15.1 \mathrm{~nm}$. $a$ and $c$ are the length of the major and minor axes, respectively. The acute orientation angle $\theta$ is formed between the major axis and the $y$ axis. $A_{0}=L_{y} L_{z}$ is the initial simulation cell area and $A_{\text {void }}$ is the cross-sectional area of the void, both of which are on the $y-z$ plane.

throughout the specimen is along the $x$ axis. The acute orientation angle $\theta$ is formed between the major axis and the $y$ axis, as illustrated in Fig. 1(b). In our initial configurations with a fixed cross-sectional area of the void on the $y$ - $z$ plane, the ellipticity $e$ varies from 0 (circular), $0.2,0.4,0.6$, to 0.8 , and the orientation angle $\theta$ varies from $0^{\circ}, 15^{\circ}, 30^{\circ}, 45^{\circ}, 60^{\circ}, 75^{\circ}$, to $90^{\circ}$. The simulation domain is $15.1 \mathrm{~nm}\left(L_{x}\right)$ by $100.1 \mathrm{~nm}\left(L_{y}\right)$ by $100.1 \mathrm{~nm}\left(L_{z}\right)$ along the $x, y$, and $z$ directions, respectively, with $\{100\}$ faces. As a result, each simulation cell contains about 12.31 millions atoms with the lattice parameter of $3.615 \AA$. The thickness of the $\mathrm{Cu}$ thin film, $L_{x}$, is chosen to reduce the effect of the film thickness on the stress-strain response [29]. To achieve a balance between the requirement of a smaller void to reduce the boundary 
effects and the need of a higher porosity to reinforce the void shape effect [31], we set the initial void area as $\pi a c=144 \pi \mathrm{nm}^{2}$, with an initial porosity of approximately $4.34 \%$ in all models. For some voids, we explore the effects of the initial porosity in Section 3.1.4.

The embedded-atom method (EAM) potential of Mishin et al. [32] is adopted for the interatomic force/energy. A parallel atomistic simulator, LAMMPS [33], is employed for all atomisitc simulations. Once a void is formed, energy minimization using a conjugate gradient algorithm is performed to attain the minimum energy configuration, followed by dynamic relaxation for 20 ps to reach an equilibrium state. Then, a uniaxial homogeneous deformation is applied along the $y$ direction at a strain rate of $10^{9} \mathrm{~s}^{-1}$, during which the virial stress is calculated every 2 ps. The effects of the loading direction and strain rate are explored in Section 3.1.3 and Section 3.1.2, respectively. In all dynamic simulations, a Velocity Verlet algorithm with a time step of 2 fs, as well as a Nosé-Hoover NPT integrator, are employed to update the atomic positions while maintaining a constant temperature of $10 \mathrm{~K}$. Such a low temperature is chosen because we are more interested in the athermal structural evolution of voids. The influence of temperature is investigated in Section 3.1.1. During the tensile loading, both $L_{x}$ and $L_{z}$ are allowed to vary to zero relevant stress components, and the porosity $f$ on the $y$ - $z$ plane at $x=L_{x} / 2$ is calculated as $A_{\text {void }} / A$, where $A_{\text {void }}$ and $A$ are the area of the void and the simulation domain measured on the same plane, respectively, as illustrated in Fig. 1(a). Simulation results are visualized using OVITO [34], an open source software in which local atomic structures and dislocations are identified by the adaptive common neighbor analysis 
(a-CNA) [35]. The Matlab code to calculate $A, f$, and the void shape can be downloaded from our website [36]. Some simulations were completed using Comet and Bridges on Extreme Science and Engineering Discovery Environment (XSEDE) [37].

\section{Results and discussion}

\subsection{Effects of cerntain variables ( $T, \dot{\varepsilon}, \beta$, and $\left.f_{0}\right)$}

In this section, we perform MD simulations to explore the effects of certain variables including the temperature $T$, the strain rate $\dot{\varepsilon}$, the loading direction angle $\beta$, and the initial porosity $f_{0}$, in the growth process of four voids: the initially circular void and the elliptic voids of $e=0.8$ with $\theta=0^{\circ}, 45^{\circ}$, and $90^{\circ}$, respectively.

\subsubsection{Temperature $T$}

For all four voids, MD simulations are performed at a constant temperature of $300 \mathrm{~K}$. The stress-strain curves for these four voids at both $10 \mathrm{~K}$ and $300 \mathrm{~K}$ are plotted in Fig. 2(a), with the snapshot of dislocation nucleation from the surface of a circular void at $300 \mathrm{~K}$ shown in Fig. 2(b). For the initially circular void and the elliptic void with $\theta=90^{\circ}$, the development of porosity $f$ with an increasing strain at both $10 \mathrm{~K}$ and $300 \mathrm{~K}$ is plotted in Fig. 2(c), with the shape evolution of the elliptic void with $\theta=90^{\circ}$ at $300 \mathrm{~K}$ shown in Fig. 2(d). It is found that (1) a higher temperature is accompanied by a lower yield stress, (2) there are noises in the snapshot shown in Fig. 2(b) which may interfere with the recognition of lattice defects, and (3) the two temperatures give very similar porosity and void shape evolution. Our find- 


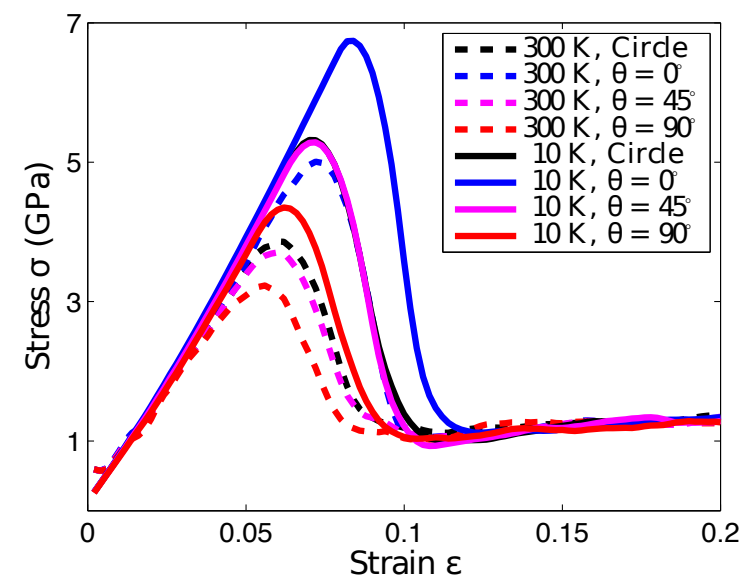

(a) Stress-strain curves

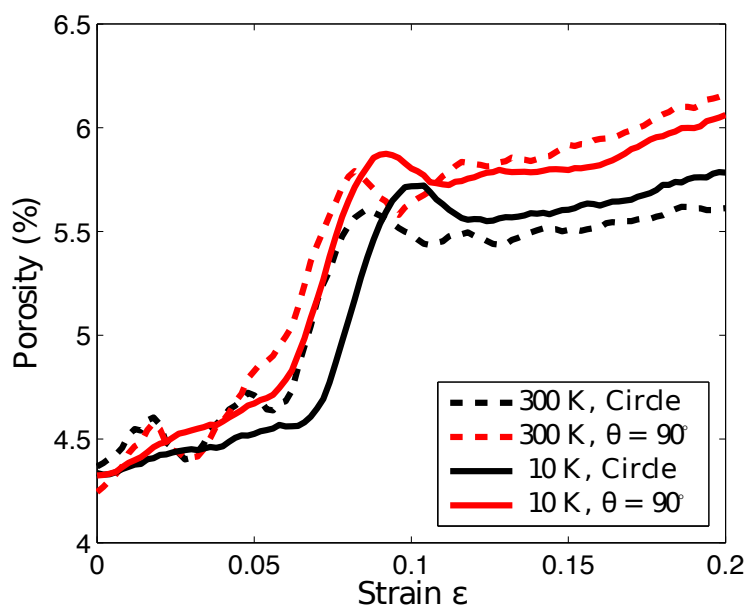

(c) Porosity-strain curves

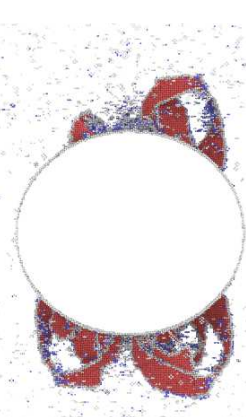

(b) Circle, $\varepsilon=0.074, T=300 \mathrm{~K}$

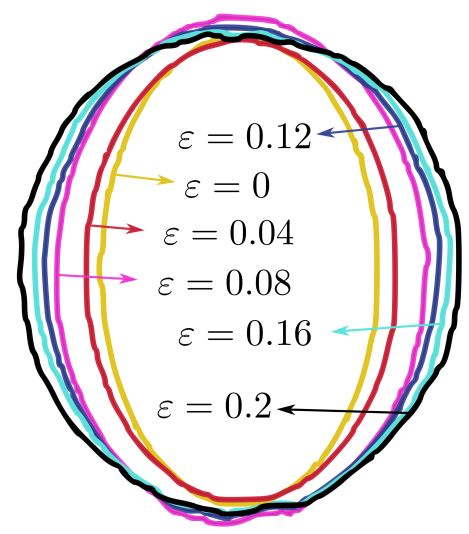

(d) $e=0.8, \theta=90^{\circ}, T=300 \mathrm{~K}$

Figure 2: Effects of the temperature $T$ in (a) the stress-strain curves, (b) the dislocation nucleation from the void surface, (c) the porosity evolution, and (d) the void shape evolution. In (b), atoms are colored by an a-CNA method [35]: red are of local HCP structure, white are the void surface, blue are of local BCC structure, while all FCC atoms are removed. The same rendering method is used throughout this paper for all atomic configurations. 
ings agree with earlier MD work by Chang et al. [18]. In the remainder of this paper, a constant temperature of $10 \mathrm{~K}$ is employed.

\subsubsection{Strain rate $\dot{\varepsilon}$}
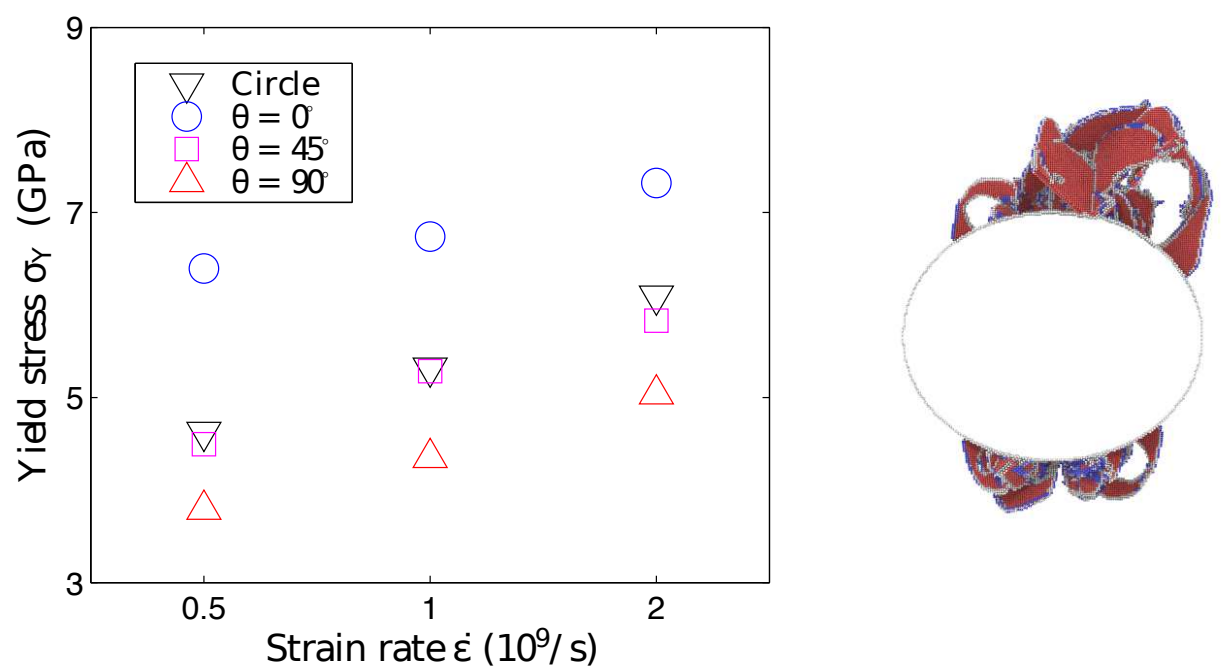

(a) Strain rate-dependent yield stress

(b) Circle, $\varepsilon=0.074, \dot{\varepsilon}=2 \times 10^{9} / \mathrm{s}$
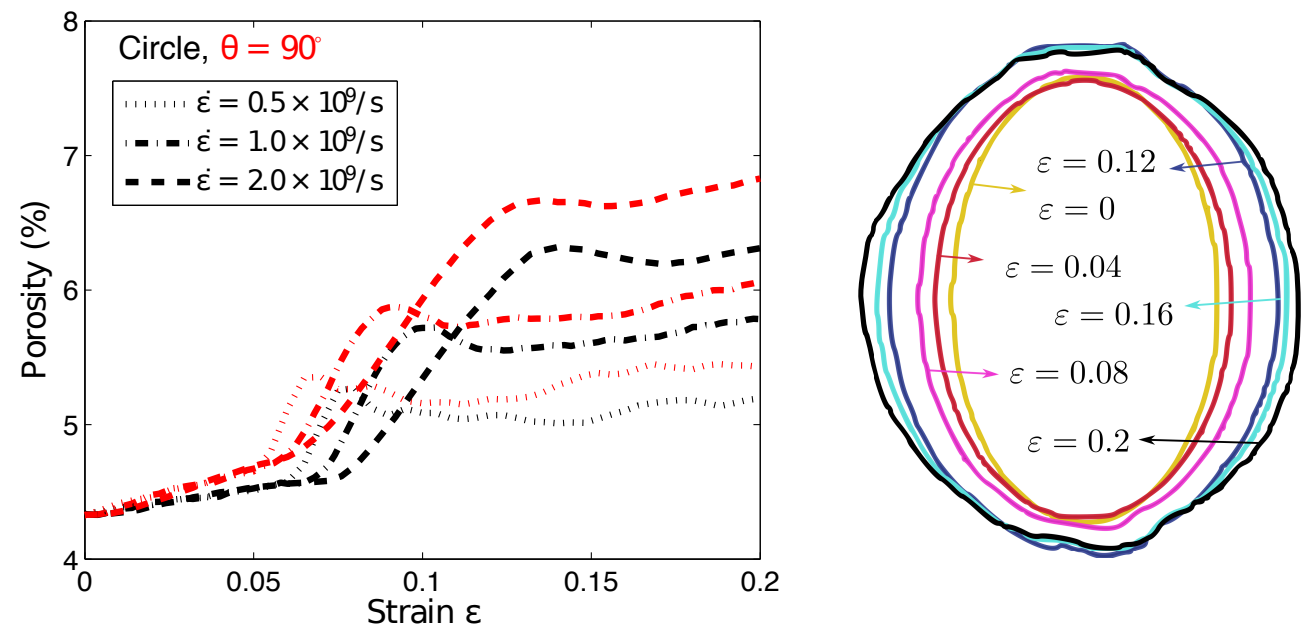

(c) Porosity-strain curves

(d) $e=0.8, \theta=90^{\circ}, \dot{\varepsilon}=2 \times 10^{9} / \mathrm{s}$

Figure 3: Effects of the strain rate $\dot{\varepsilon}$ in (a) the yield stress, (b) the dislocation nucleation from the void surface, (c) the porosity evolution, and (d) the void shape evolution. 
For all four voids, MD simulations are performed at three different strain rates, $5 \times 10^{8} \mathrm{~s}^{-1}, 10^{9} \mathrm{~s}^{-1}$, and $2 \times 10^{9} \mathrm{~s}^{-1}$, respectively. The yield stresses for these four voids at different strain rates are plotted in Fig. 3(a), with the snapshot of dislocation nucleation from the surface of a circular void at a strain rate of $2 \times 10^{9} \mathrm{~s}^{-1}$ shown in Fig. 3(b). For the initially circular void and the elliptic void with $\theta=90^{\circ}$, the development of porosity $f$ with an increasing strain at different strain rates is plotted in Fig. 3(c); the shape evolution of the elliptic void with $\theta=90^{\circ}$ at a strain rate of $2 \times 10^{9} \mathrm{~s}^{-1}$ is shown in Fig. 3(d). It is found that (1) a higher strain rate is accompanied by a higher yield stress, (2) at the same strain, a higher strain rate gives a larger number of dislocation at the void surface, (3) a higher strain rate corresponds to a larger porosity at the same strain, and (4) the effects of the void orientation angle $\theta$ are reduced with a higher strain rate. The first three findings agree with the earlier MD work by $\mathrm{Xu}$ et al. [23]. In the remainder of this paper, a strain rate of $10^{9} \mathrm{~s}^{-1}$ is adopted.

\subsubsection{Loading direction angle $\beta$}

For all four voids, MD simulations are performed with the simulation cell uniformly deformed along the directions which form angles $\beta=30^{\circ}$ and $60^{\circ}$ with the $y$ direction, respectively, as illustrated in Fig. 4(a). The yield stresses for these four voids in cases of four different angles $\beta=0^{\circ}, 30^{\circ}, 60^{\circ}$, and $90^{\circ}$ are plotted in Fig. 4(b). For the initially circular void and the elliptic void with $\theta=90^{\circ}$, the development of porosity $f$ with an increasing strain in cases of $\beta=0^{\circ}, 30^{\circ}$, and $60^{\circ}$ is plotted in Fig. 4(c); the shape evolution of the elliptic void with $\theta=90^{\circ}$ in the case of $\beta=30^{\circ}$ is shown in Fig. $4(\mathrm{~d})$. It is found that the yield stress is affected by the loading direction angle $\beta$. 


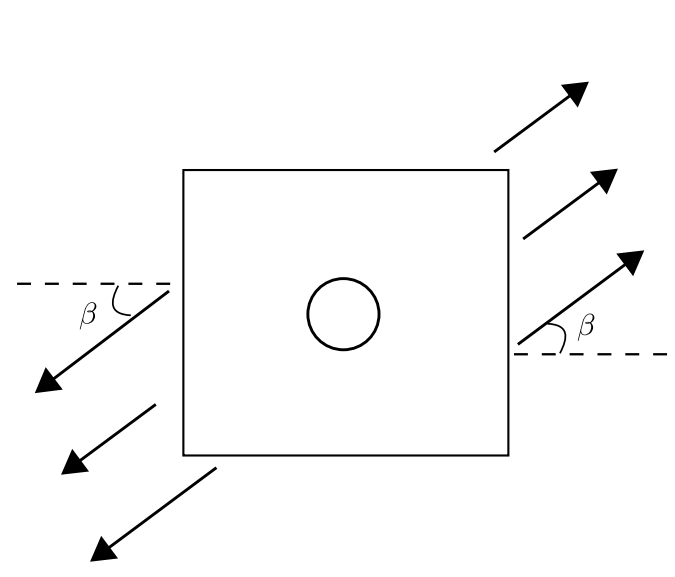

(a)

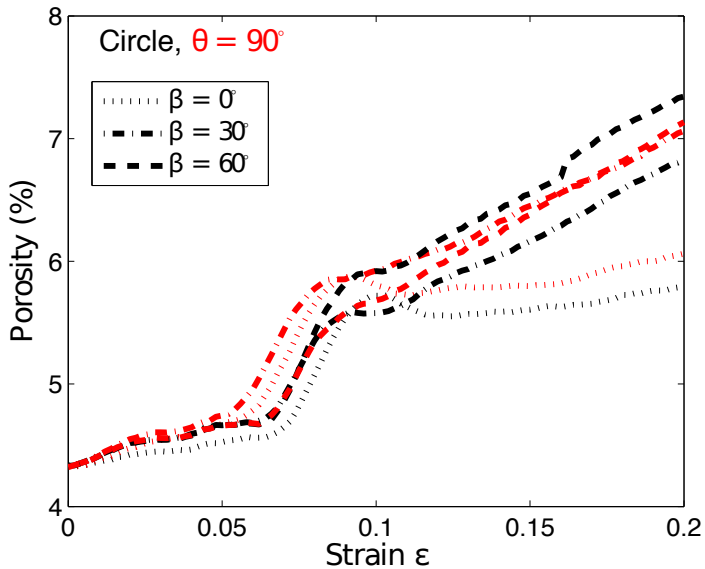

(c) Porosity-strain curves

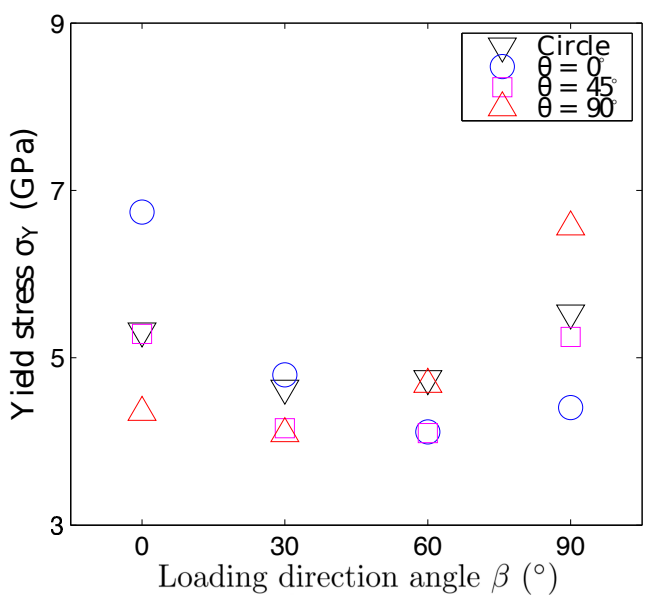

(b) Loading direction-dependent yield stress

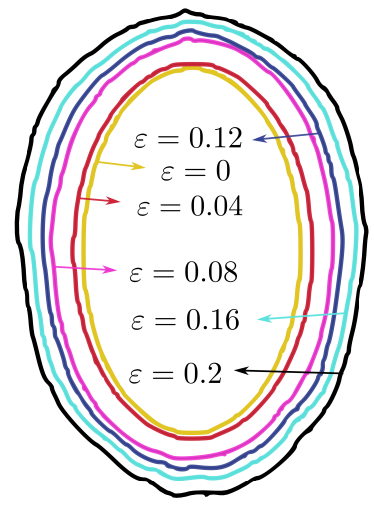

(d) $e=0.8, \theta=90^{\circ}, \beta=30^{\circ}$

Figure 4: Effects of the loading direction angle $\beta$, illustrated in (a), in (b) the yield stress, (c) the porosity evolution, and (d) the void shape evolution.

In the remainder of this paper, a loading direction angle $\beta=0^{\circ}$ is employed.

\subsubsection{Initial porosity $f_{0}$}

For all four voids, MD simulations are performed in cases of four different initial void area $A_{0}: 144 \pi \mathrm{nm}^{2}, 81 \pi \mathrm{nm}^{2}, 36 \pi \mathrm{nm}^{2}$, and $9 \pi \mathrm{nm}^{2}$; the cor- 


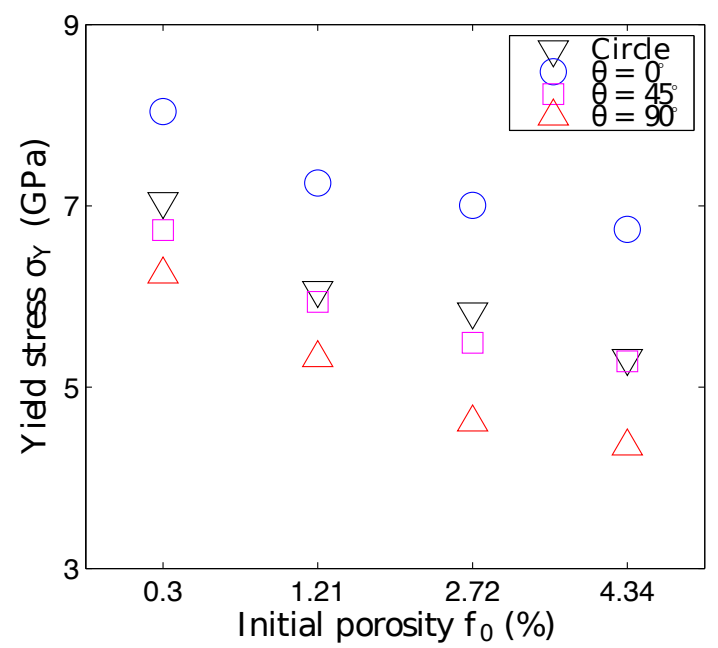

(a) Initial porosity-dependent yield stress

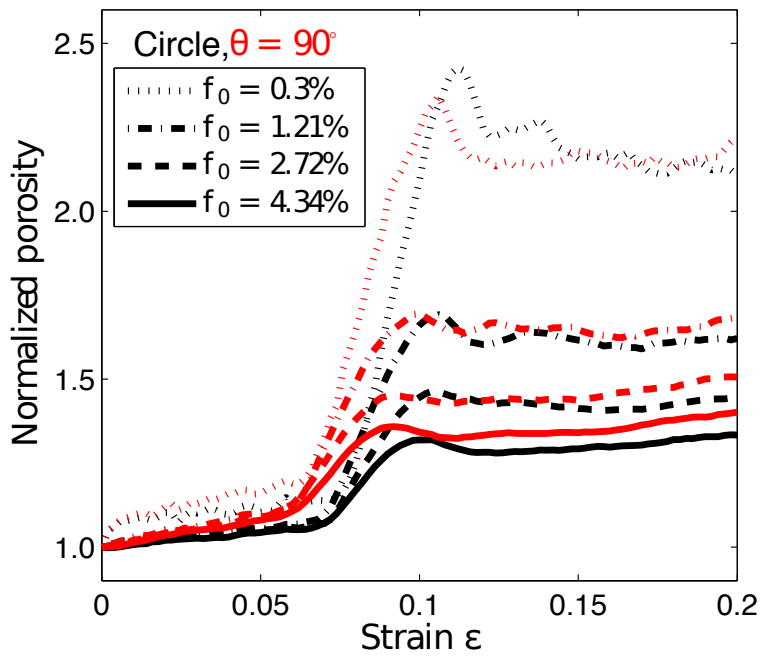

(c) Normalized porosity-strain curves

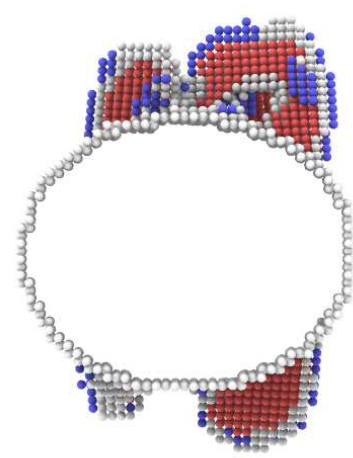

(b) Circle, $\varepsilon=0.074, f_{0}=0.3 \%$

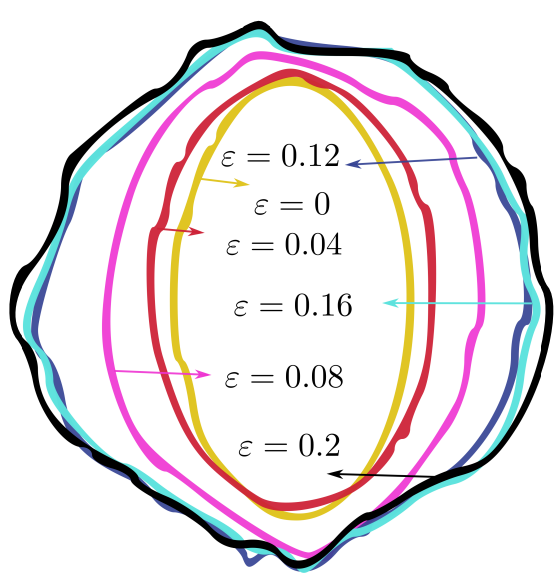

(d) $e=0.8, \theta=90^{\circ}, f_{0}=0.3 \%$

Figure 5: Effects of the initial porosity $f_{0}$ in (a) the yield stress, (b) the dislocation nucleation from the void surface, (c) the porosity evolution, and (d) the void shape evolution.

responding initial porosity $f_{0}$ is $4.34 \%, 2.72 \%, 1.21 \%$, and $0.3 \%$. The yield stresses for all these voids are plotted in Fig. 5(a), with the snapshot of dislocation nucleation from the surface of a circular void with initial $f=0.3 \%$ 
shown in Fig. 5(b). For the initially circular void and the elliptic void with $\theta=90^{\circ}$, the development of the normalized porosity $f / f_{0}$ with an increasing strain in cases of different initial porosity is plotted in Fig. 5(c); the shape evolution of the elliptic void with $\theta=90^{\circ}$ and $f_{0}=0.3 \%$ is shown in Fig. 5(d). It is found that (1) a larger initial porosity is accompanied by a lower yield stress, (2) at the same strain, a smaller initial porosity gives a smaller number of dislocation at the void surface due to the reduced initial void area, (3) a smaller initial porosity increases the normalized porosity at the same strain, and (4) the effects of the void orientation angle $\theta$ are more pronounced with a larger initial porosity. The first three findings agree with earlier MD work by Potirniche et al. [17]. In the remainder of this paper, an initial void area $A_{0}=144 \pi \mathrm{nm}^{2}$, corresponding to an initial porosity $f_{0}=4.34 \%$, is employed.

\subsection{Dislocation nucleation}

Figure 6 presents a series of snapshots of dislocation nucleation from the surface of a circular void (ellipticity $e=0$ ) at different strain $\varepsilon$. The variation of $\theta$ takes no effect in this case. When the applied strain is small $(\varepsilon<0.07)$, both the simulation cell and void expand elastically, free of dislocations. At $\varepsilon=0.07$, dislocations begin to nucleate from the top side of the void and

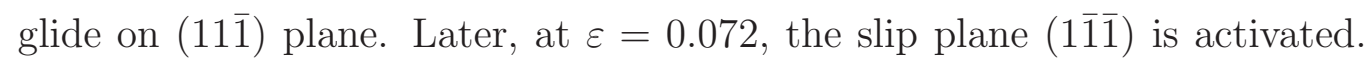
At larger strains of 0.074 and 0.076 , dislocations begin to glide on (111) and (111) planes, respectively. Preivous MD simulations of a $3-\mathrm{D}$ void in $\mathrm{Cu}$ also showed that while all four $\{111\}$ planes are equivalent in terms of the Schmid factor, dislocations are usually initiated on one slip plane, followed by activation of others [19]. At an even larger strain of 0.078, some 


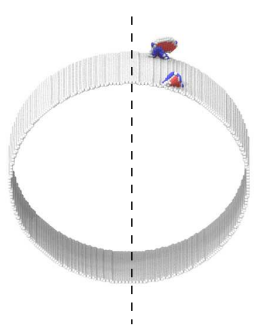

(a) $\varepsilon=0.07$

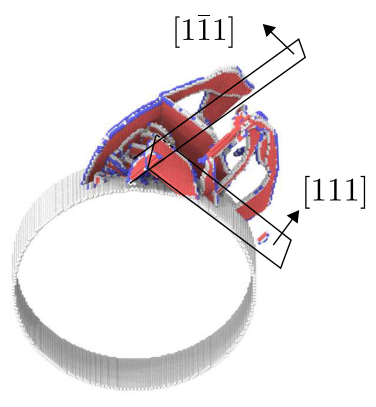

(d) $\varepsilon=0.076$

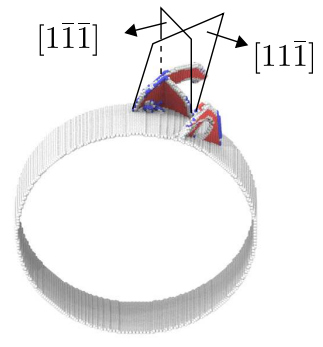

(b) $\varepsilon=0.072$

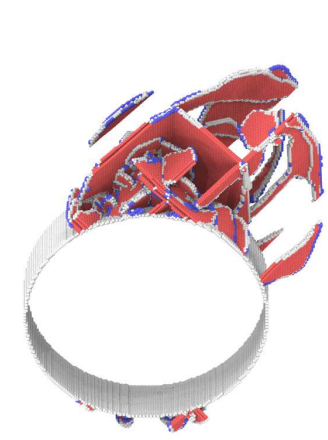

(e) $\varepsilon=0.078$

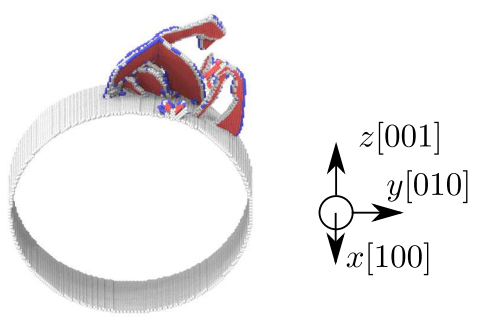

(c) $\varepsilon=0.074$

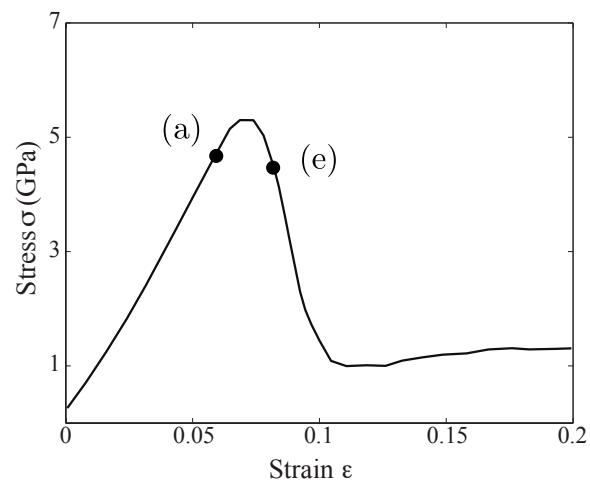

(f) Stress-strain curve

Figure 6: (a-e) Snapshots of dislocation nucleation from the surface of a circular void. Note that dislocations pass through the PBCs normal to the $x$ direction to reconnect themselves, forming a series of dislocation loops. The line of symmetry of the void with respect to the $z$ direction is marked by a dashed line in (a). The strains corresponding to (a) and (e) are labeled in the stress-strain curve in (f).

dislocations nucleating from the top side cross the PBCs normal to the $z$ axis; meanwhile, some other dislocations are initiated from the bottom side of the void. Overall, a majority of dislocations nucleate at the top/bottom sides of the void, a reflection of the fact that these two sites have the strongest stress concentrations in a model subject to a tensile loading along the $y$ direction.

For an initially elliptic void $(e>0)$, both the sequence of slip plane activation and the initial dislocation emission sites vary with $e$ and $\theta$. A series 


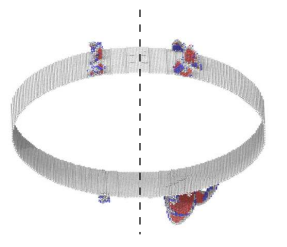

(a) $\varepsilon=0.084$

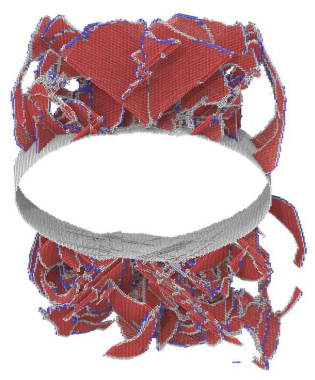

(d) $\varepsilon=0.09$

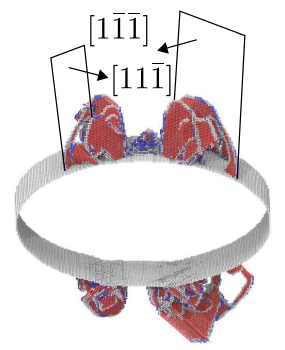

(b) $\varepsilon=0.086$

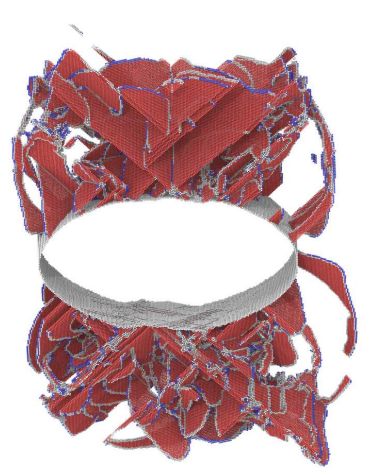

(e) $\varepsilon=0.092$

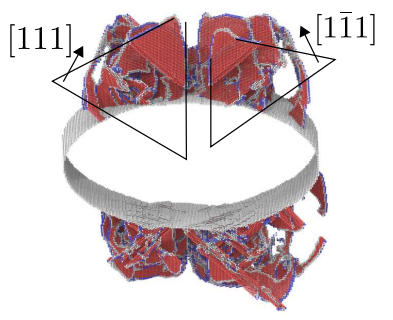

$\stackrel{\substack{z[001] \\ \Psi_{x[100]}}[010]}{\longrightarrow}$

(c) $\varepsilon=0.088$

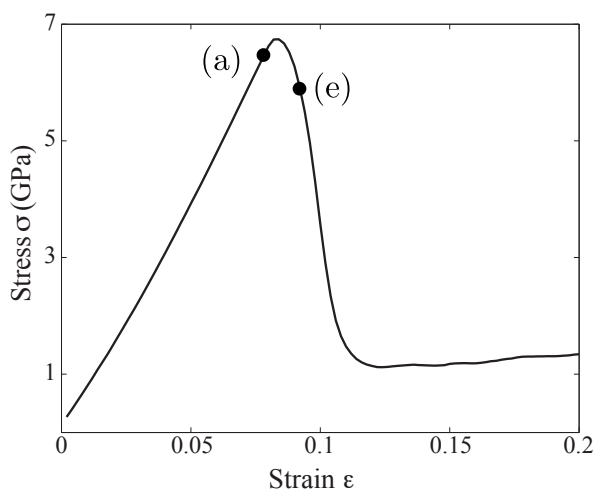

(f) Stress-strain curve

Figure 7: (a-e) Snapshots of dislocation nucleation from the surface of a void of $e=0.8$ with $\theta=0^{\circ}$. The minor axis of the void is marked by a dashed line in (a). The strains corresponding to (a) and (e) are labeled in the stress-strain curve in (f).

of atomic configurations of dislocation nucleation from a void with $e=0.8$ are shown in Fig. $7\left(\theta=0^{\circ}\right)$, Fig. $8\left(\theta=45^{\circ}\right)$, and Fig. $9\left(\theta=90^{\circ}\right)$. In Fig. 7 ,

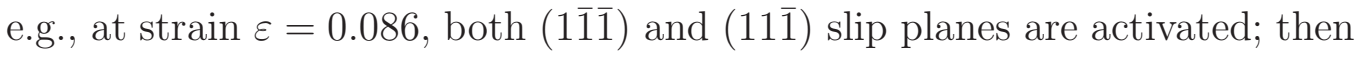
at a larger strain of $\varepsilon=0.088$, dislocations start to glide on the other two slip planes, (111) and (111) planes. In none of these cases are all $\{111\}$ slip planes activated simultaneously. Compared with an initially circular void, dislocations begin to nucleate at both top/bottom sides of an initially elliptic void under the same strain; furthermore, the initial dislocation emission sites 


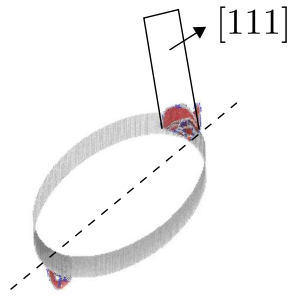

(a) $\varepsilon=0.07$

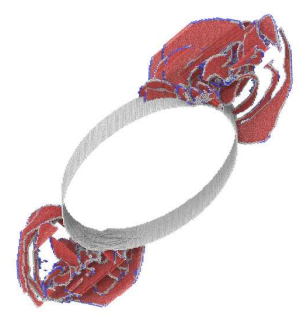

(d) $\varepsilon=0.076$

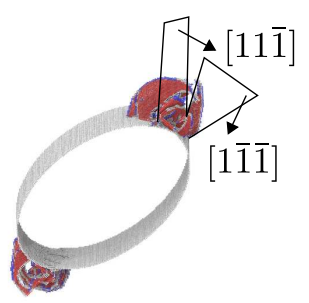

(b) $\varepsilon=0.072$

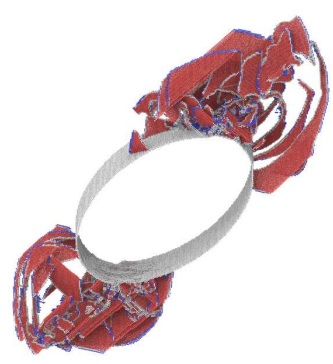

(e) $\varepsilon=0.078$

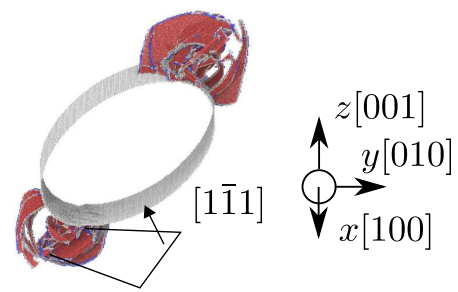

(c) $\varepsilon=0.074$

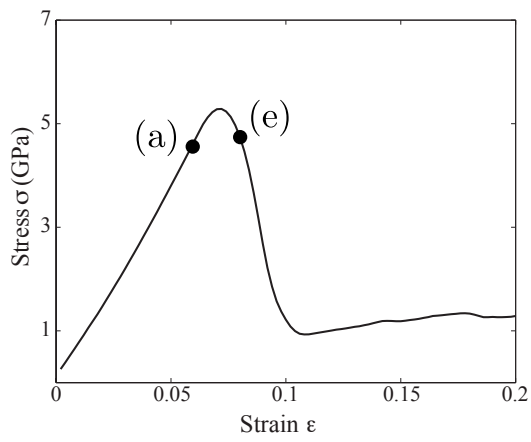

(f) Stress-strain curve

Figure 8: (a-e) Snapshots of dislocation nucleation from the surface of a void of $e=0.8$ with $\theta=45^{\circ}$. The major axis of the void is marked by a dashed line in (a). The strains corresponding to (a) and (e) are labeled in the stress-strain curve in (f).

on the surface of an initially elliptic void are farther from and closer to the line of symmetry with respect to the $z$ axis, when $\theta=0^{\circ}$ and $90^{\circ}$, respectively. This change of the dislocation nucleation sites with the ellipticity $e$ was also reported by Cui and Chen [29] in Al. In the case of $\theta=45^{\circ}$, the distance between the initial dislocation nucleation sites and the major axis is almost the same with that for an initially circular void, as shown in Fig. 6 and Fig. 8. This similarity corresponds to the fact that their yield points are close to each other, as will be shown in Section 3.4. 


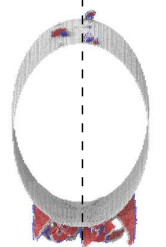

(a) $\varepsilon=0.052$

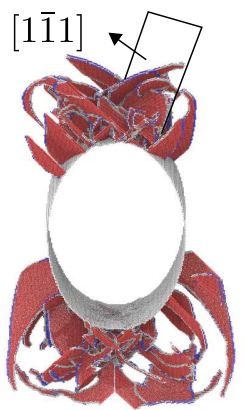

(d) $\varepsilon=0.058$

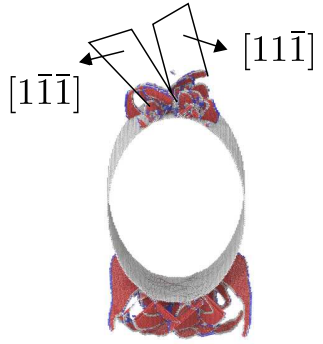

(b) $\varepsilon=0.054$

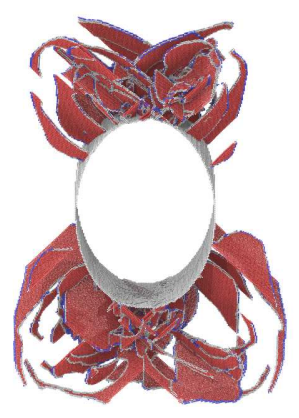

(e) $\varepsilon=0.06$

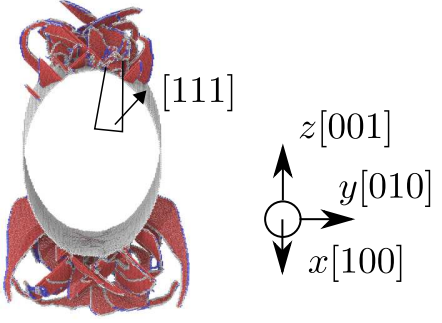

(c) $\varepsilon=0.056$

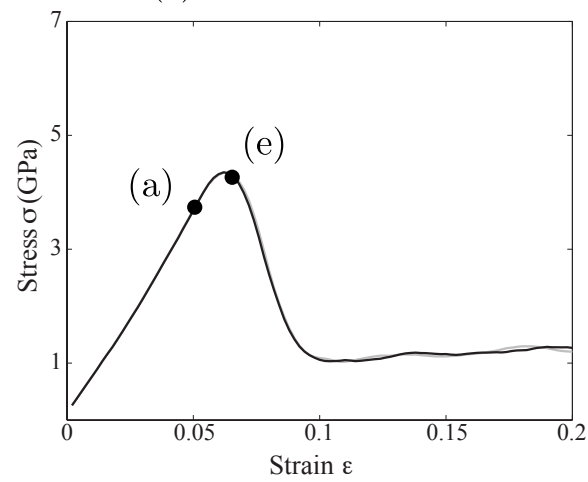

(f) Stress-strain curve

Figure 9: (a-e) Snapshots of dislocation nucleation from the surface of a void of $e=0.8$ with $\theta=90^{\circ}$. The major axis of the void is marked by a dashed line in (a). The strains corresponding to (a) and (e) are labeled in the stress-strain curve in (f).

\subsection{Void evolution}

Figure 10(a) presents the evolution of the porosity $f$ with an increasing strain $\varepsilon$ for voids that begin with a circular shape $(e=0)$ and an elliptic shape $(e=0.8)$ with $\theta=0^{\circ}, 45^{\circ}$, and $90^{\circ}$, respectively. Three regimes of void growth are identified. In regime I, at a small $\varepsilon, f$ increases relatively slowly with that for $\theta=90^{\circ}$ being the fastest among the four cases. Then, once the dislocations begin to nucleate, e.g., at $\varepsilon=0.07$ for a circular void, as marked by the vertical arrows in Fig. 10(a), regime II is initiated. In this regime, $f$ starts to grow much faster due to the dislocation nucleation 

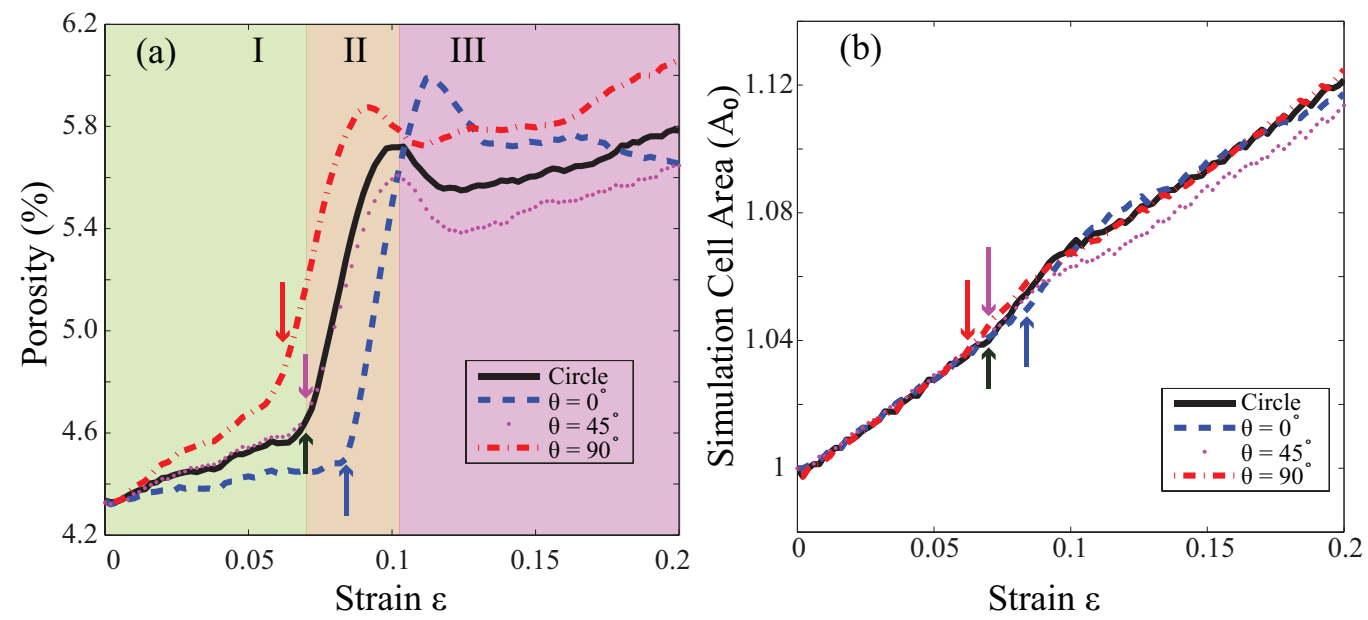

Figure 10: (a) Porosity $f$ and (b) simulation cell area $A$ as a function of strain $\varepsilon$ for void of a circular shape $(e=0)$ and of an elliptic shape $(e=0.8)$ with three different orientation angle $\theta . A_{0}$ is the initial simulation cell area on the $y$ - $z$ plane. The vertical arrows mark initial dislocation emission. Based on the circular void, three regimes of porosity evolution are distinguished using different background colors.

and migration from the void surface that push surrounding atoms outwards. Later, at $\varepsilon \approx 0.1$ for a circular void, regime III starts in which $f$ decreases slightly before continuing growing, yet at a lower rate than that in regimes I and II. This can be explained by the saturation of dislocation in which some dislocations push atoms inwards to void. Among the four cases, the elliptic void with $\theta=0^{\circ}$ has the highest onset strain and porosity for regime III, and is the only case in which the porosity $f$ keeps declining after the peak. Further analysis confirms that the decrease in $f$ is due to a declining void area $A_{\text {void, }}$ even when the simulation cell area $A$ continues increasing in regime III. This is probably because that, compared with the other three cases, the top/bottom sides of the elliptic void with $\theta=0^{\circ}$ have a longer distance 

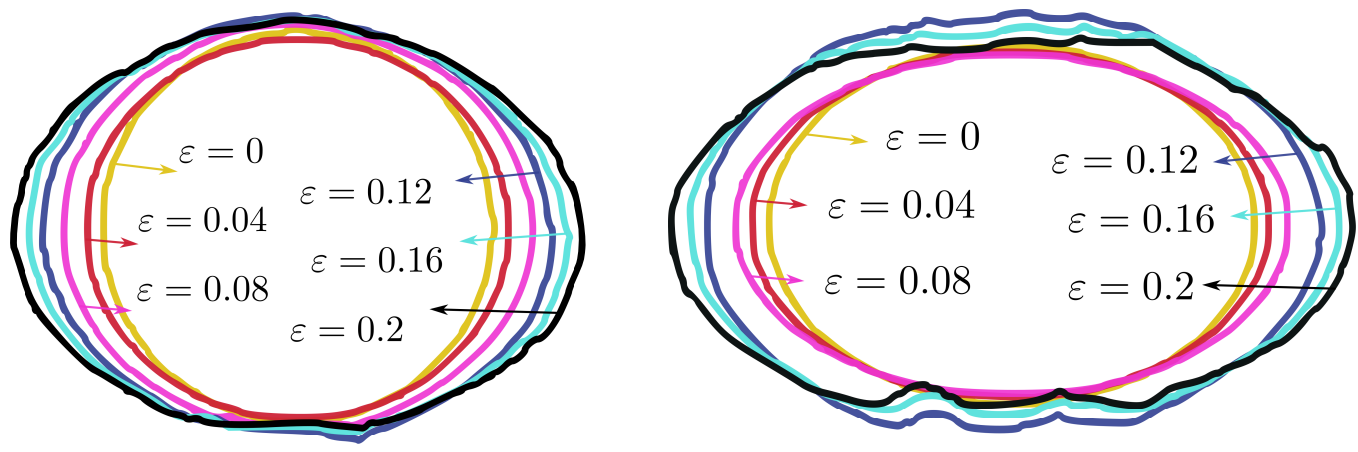

(a) Circle, $e=0$
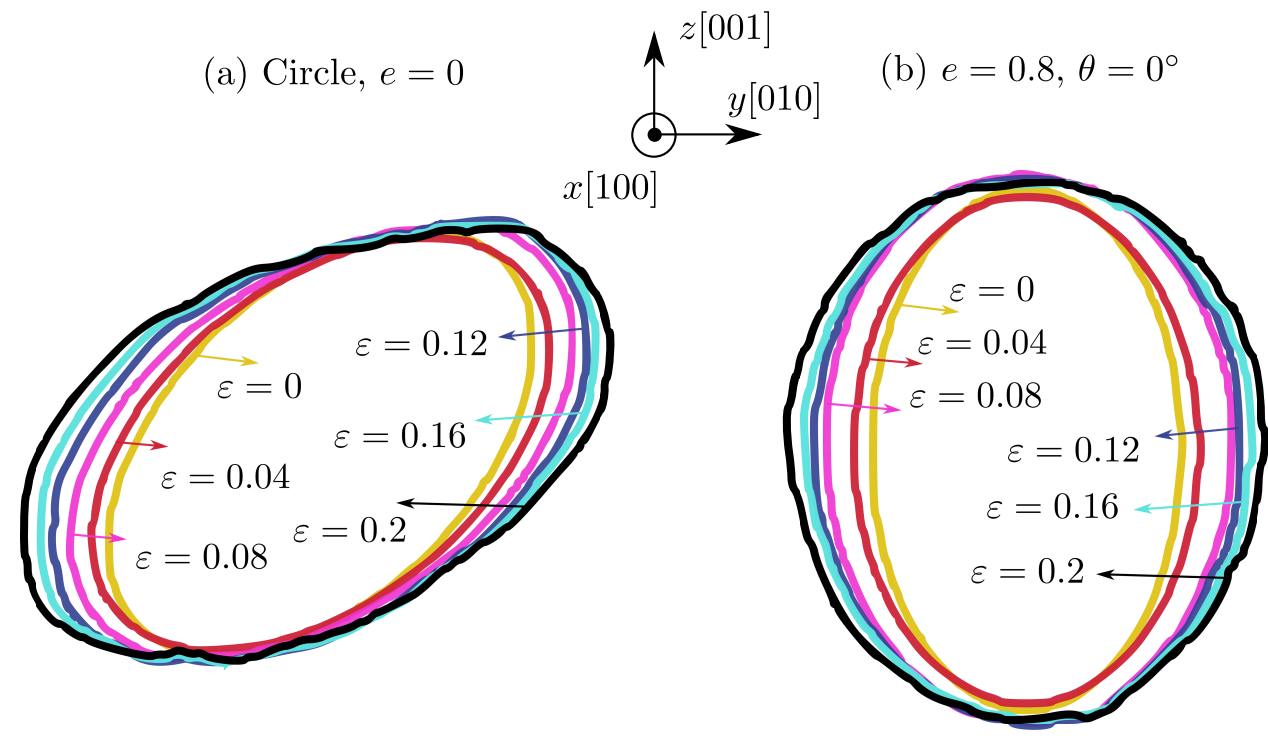

(c) $e=0.8, \theta=45^{\circ}$

(d) $e=0.8, \theta=90^{\circ}$

Figure 11: Shape evolution of a void with (a) a circular shape with $e=0$ and an elliptic shape with $e=0.8$ when (b) $\theta=0^{\circ}$, (c) $\theta=45^{\circ}$, and (d) $\theta=90^{\circ}$. Void shape measured at different strain $\varepsilon$ is distinguished by different colors.

away from the periodic cell boundaries normal to the $z$ axis, which allows more room for the dislocation and void development prior to the saturation; such a high dislocation density facilitates pushing atoms toward void, and so reducing the porosity.

Prior to the initial dislocation emission, which is marked by arrows in 
Fig. 10(b), the simulation cell area $A$ in the four cases increase linearly with $\varepsilon$ at the same pace, as a result of the Poisson's ratio of $\mathrm{Cu}$ at the elastic stage $\nu=0.34$. Then, at larger strains, in the presence of dislocations, the simulation cell area $A$ in the four cases are no longer the same, with $A$ being the smallest, i.e., $\nu$ being the largest, for the elliptic void with $\theta=45^{\circ}$. This quasi-linear evolution of $A$ with $\varepsilon$ indicates that the change in porosity $f$ is mainly due to the change of the void area $A_{\text {void }}$.

The outline evolution of these four voids is displayed in Fig. 11. Take the circular void as an example. In regime I ( $\varepsilon=0$ and 0.04$)$, the void size along the $y$ axis increases while that along the lateral $z$ direction shrinks, in the same way as the simulation cell. In regime II $(\varepsilon=0.08)$, the void grows along both the $y$ and $z$ axes, with the latter at a slower pace, as a result of the dislocation nucleation and migration. This bi-directional void growth is in agreement with the fast increase of the porosity $f$ in this regime. In regime III $(\varepsilon=0.12,0.16$, and 0.2$)$, the void size increases along the $y$ axis only, while that along the $z$ axis remains invariant. Moreover, the void surface becomes irregular, non-smooth. The outline evolution of the elliptic voids follows a similar pattern as the circular one, expect that for $\theta=0^{\circ}$, while continuing expanding along the $y$ direction, the void begins to shrink along the $z$ axis again, corresponding to a decreasing porosity $f$ after the peak, as shown in Fig. 10(a). Note that no cleavage or voids coalescence across the PBCs is observed up to $\varepsilon=0.2$.

\subsection{Stress-strain response}

In this section, we summarize the stress-strain curves for different initial ellipticity $e(0 \sim 0.8)$ and initial orientation angle $\theta\left(0^{\circ} \sim 90^{\circ}\right)$ in Fig. 12 , 


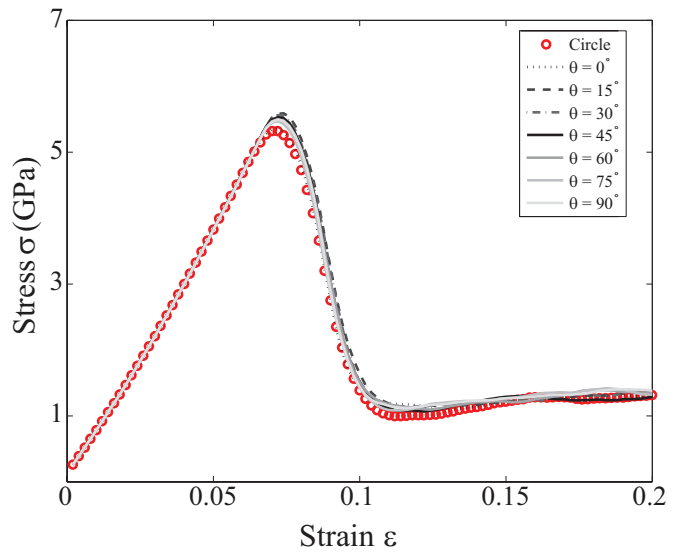

(a) $e=0.2$

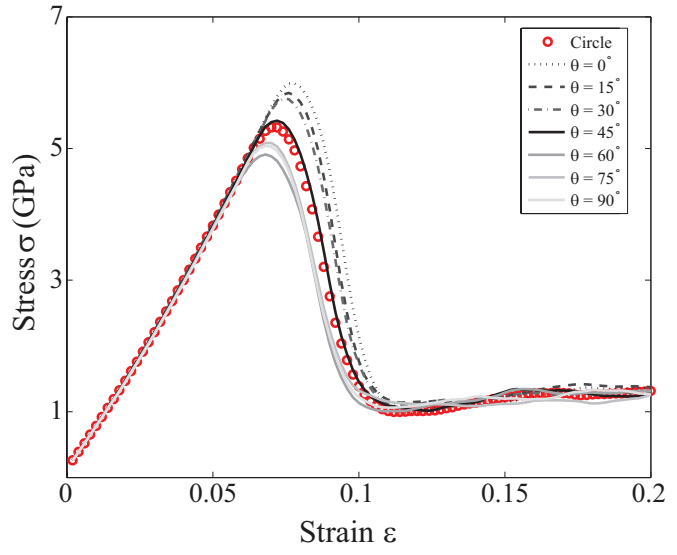

(c) $e=0.6$

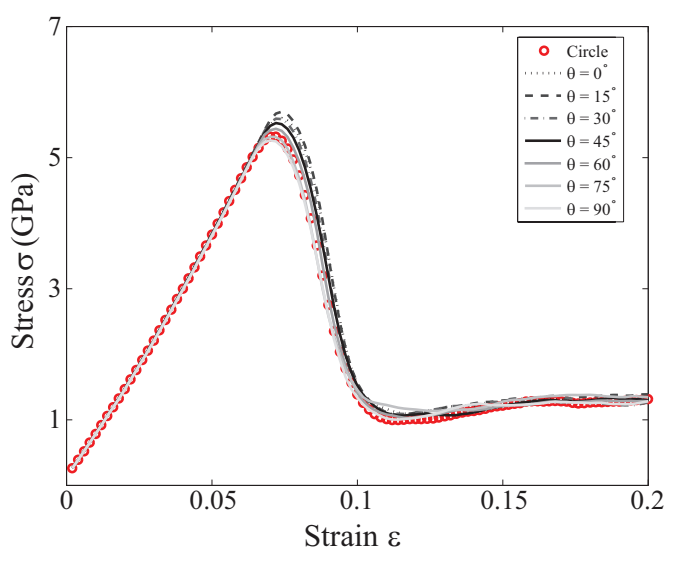

(b) $e=0.4$

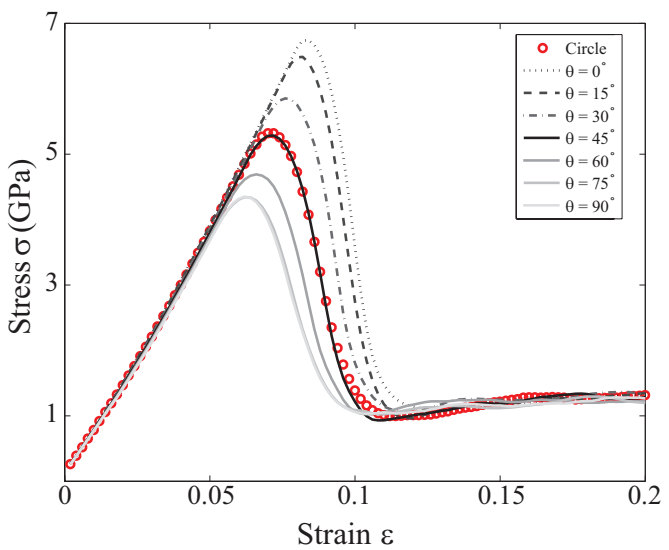

(d) $e=0.8$

Figure 12: Stress-strain curves in the cases of (a) $e=0.2$, (b) $e=0.4$, (c) $e=0.6$, and (d) $e=0.8$ for different orientation angle $\theta$.

in all of which the case of a circular void is used as a reference. It is found that for different $e$, the degree of the influence of the orientation angle $\theta$ on the stress-strain response is different. For the same $\theta$, varying $e$ alters the stress-strain curve. To better illustrate these effects, we show a plot of the yield stress $\sigma_{\mathrm{Y}}$ and the strain at the yield point $\varepsilon_{\mathrm{Y}}$ as a function of $e$ 


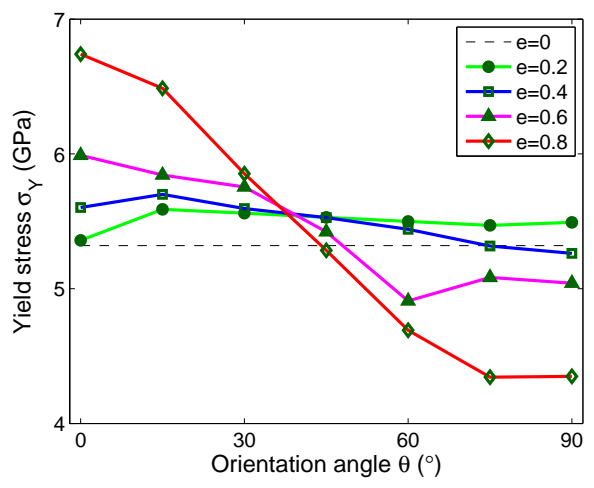

(a) Yield stress

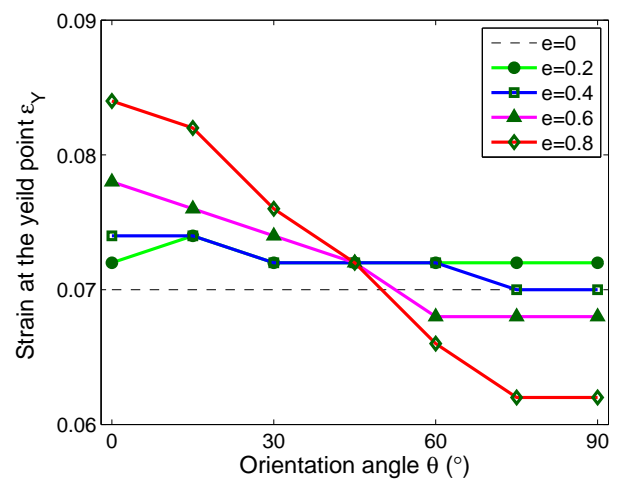

(b) Strain at the yield point

Figure 13: (a) Yield stress and (b) strain at the yield point as a function of both ellipticity $e$ and orientation angle $\theta$.

and $\theta$ in Fig. 13, where the yield point corresponds to the initial dislocation emission. We remark that in all simulations, dislocations begin to nucleate from the void surface prior to the maximum stress. Compared with the circular void, the difference between $\varepsilon_{\mathrm{Y}}$ and the strain corresponding to the maximum stress is larger when $\theta=90^{\circ}$ and smaller when $\theta=0^{\circ}$, as shown in subfigures (f) of Fig. 6, Fig. 7, and Fig. 9; similar results were reported by Cui and Chen [29] in Al. Note that both the slope of the stress-strain curve prior to the yield point and the flow stress remain the same for different $e$ and $\theta$, indicating that the Young's modulus at the elastic stage is invariant with respect to these two factors.

From Fig. 13, the general trend is that a larger $\theta$ would result in smaller $\sigma_{\mathrm{Y}}$ and $\varepsilon_{\mathrm{Y}}$, and the effect of $\theta$ is the most pronounced for the largest $e$. In the meantime, the effect of $e$ on the stress-strain response depends on $\theta$. Specifically, for a small orientation angle, e.g., $\theta \leq 30^{\circ}$, a larger $e$ is 
accompanied by higher $\sigma_{\mathrm{Y}}$ and $\varepsilon_{\mathrm{Y}}$; for a large angle, e.g., $\theta \geq 60^{\circ}$, a larger $e$ leads to lower $\sigma_{\mathrm{Y}}$ and $\varepsilon_{\mathrm{Y}}$; for an intermediate $\theta$, e.g, $45^{\circ}$, the variation of $e$ takes little effect on the stress-strain response. This is because on the one hand, dislocations alway start to nucleate from the top and bottom sides of the void, where the largest stress concentrations are present; on the other hand, for elliptic voids $(e>0)$, a larger $\theta$ results in a larger curvature of the void's cross-sectional area on the $y$ - $z$ plane at its top and bottom, which further intensifies the stress concentrations at these sites and thus advances the yield point by lowering both $\sigma_{\mathrm{Y}}$ and $\varepsilon_{\mathrm{Y}}$. From the perspective of damage mechanics, in the case of a large $\theta$, the effective stress along the $y$ axis is increased by a larger cross-sectional area of the void on the mid $x-z$ plane [38], then a smaller strain is needed to yield the material, in agreement with our MD simulation results.

\section{Conclusion}

In this work, MD simulations with millions of atoms are employed to study the nanovoid growth in FCC Cu thin film with emphasis on the effects

of the initial void ellipticity $e$ and the initial orientation angle $\theta$ - two important factors dictating the void geometry. Results are summarized as follows:

1. The initial void geometry not only alters the yield stress $\sigma_{\mathrm{Y}}$ and the strain at the yield point $\varepsilon_{\mathrm{Y}}$, from a macroscopic perspective, but also affects the dislocation nucleation and the void evolution process, from a nanoscopic perspective. The effects of the initial void geometry are more pronounced in cases of a lower strain rate and/or a larger initial 
porosity.

2. In all cases, the four $\{111\}$ slip planes in FCC system, which are equivalent in terms of the Schmid factor, are activated at different strains. The activation sequence of the four slip planes varies with $e$ and $\theta$. An elliptic void with $\theta=0^{\circ}$ and $90^{\circ}$ has a greater and lesser distance between the line of symmetry of the void along the vertical direction and the initial dislocation emission sites, respectively, than the circular void.

3. In terms of the void evolution with the applied strain, three regimes are identified: in regime I, no dislocation is formed, and the porosity increases slowly; in regime II, dislocations start to nucleate from the void surface, pushing the atoms outwards and resulting in a much higher rate of porosity increasing; in regime III, the porosity variation is at a lower rate than that in regime II, due to the saturation of dislocation. In addition, in regime III, the void surface becomes irregular, nonsmooth and in some cases begins to shrink along the direction normal to that of tension, reducing the void size even when the simulation cell is expanding. The porosity and the onset strains for the three regimes, as well as the simulation cell area evolution after the yield point, vary as a function of both $e$ and $\theta$.

4. In terms of the stress-strain response, the effect of $\theta$ is more pronounced for a larger $e$. The general trend is that a larger $\theta$ results in lower $\sigma_{\mathrm{Y}}$ and $\varepsilon_{\mathrm{Y}}$. For a small angle, e.g., $\theta \leq 30^{\circ}$, an increasing $e$ is accompanied by higher $\sigma_{\mathrm{Y}}$ and $\varepsilon_{\mathrm{Y}}$; for a large angle, e.g., $\theta \geq 60^{\circ}$, a larger $e$ leads to lower $\sigma_{\mathrm{Y}}$ and $\varepsilon_{\mathrm{Y}}$; for an intermediate $\theta$, e.g., $45^{\circ}$, the variation of $e$ takes 
little effect on the stress-strain response. The slope of the stress-strain curve prior to the yield point and the flow stress remain the same for different $e$ and $\theta$.

Our work suggests that besides the porosity, the continuum model needs to incorporate the effects of the initial void ellipticity and the initial void orientation angle in a direct manner, if it is to be applied to nanoscale damage progression. Future work includes using 3-D models to study the influence of the void front curvature and to elucidate the convergence of a void with an increasing ellipticity to a crack [39].

\section{Acknowledgment}

This work used the Extreme Science and Engineering Discovery Environment (XSEDE), which is supported by National Science Foundation grant number ACI-1053575.

\section{References}

[1] P. G. Manusmare, H. P. Leighly, Void-strengthening in aluminum and its nature, Acta Metall. 24 (1976) 1047-1052.

[2] R. Nakai, K. Yabuuchi, S. Nogami, A. Hasegawa, The effect of voids on the hardening of body-centered cubic Fe, J. Nucl. Mater. 471 (2016) 233-238.

[3] J. C. Crone, L. B. Munday, J. Knap, Capturing the effects of free surfaces on void strengthening with dislocation dynamics, Acta Mater. 101 (2015) 40-47. 
[4] L. Xiong, S. Xu, D. L. McDowell, Y. Chen, Concurrent atomisticcontinuum simulations of dislocationvoid interactions in fec crystals, Int. J. Plast. 65 (2015) 33-42.

[5] T. Tang, S. Kim, M. F. Horstemeyer, Molecular dynamics simulations of void growth and coalescence in single crystal magnesium, Acta Mater. 58 (2010) 4742-4759.

[6] E. Lin, L. Niu, H. Shi, Z. Duan, Molecular dynamics study on the nanovoid growth and coalescence at grain boundary, Sci. China Phys. Mech. Astron. 55 (2011) 86-93.

[7] S. Z. Xu, Z. M. Hao, Y. Q. Su, W. J. Hu, Y. Yu, Q. Wan, Atomic collision cascades on void evolution in vanadium, Radiat. Eff. Def. Solids 167 (2012) 12-25.

[8] Y. Chen, K. Y. Yu, Y. Liu, S. Shao, H. Wang, M. A. Kirk, J. Wang, X. Zhang, Damage-tolerant nanotwinned metals with nanovoids under radiation environments, Nature Comm. 6 (2015) 7036.

[9] M. F. Horstemeyer, M. M. Matalanis, A. M. Sieber, M. L. Botos, Micromechanical finite element calculations of temperature and void configuration effects on void growth and coalescence, Int. J. Plast. 16 (2000) 979-1015.

[10] S. K. Yerra, C. Tekog̃lu, F. Scheyvaerts, L. Delannay, P. Van Houtte, T. Pardoen, Void growth and coalescence in single crystals, Int. J. Solids Struct. 47 (2010) 1016-1029. 
[11] G. P. Potirniche, J. L. Hearndon, M. F. Horstemeyer, X. W. Ling, Lattice orientation effects on void growth and coalescence in fcc single crystals, Int. J. Plast. 22 (2006) 921-942.

[12] S. Ha, K. Kim, Void growth and coalescence in f.c.c. single crystals, Int. J. Mech. Sci. 52 (2010) 863-873.

[13] U. Borg, C. F. Niordson, J. W. Kysar, Size effects on void growth in single crystals with distributed voids, Int. J. Plast. 24 (2008) 688-701.

[14] V. Tvergaard, Void shape effects and voids starting from cracked inclusion, Int. J. Solids Struct. 48 (2011) 1101-1108.

[15] X. Deng, W. Zhu, Y. Zhang, H. He, F. Jing, Configuration effect on coalescence of voids in single-crystal copper under shock loading, Comput. Mater. Sci. 50 (2010) 234-238.

[16] S. Traiviratana, E. M. Bringa, D. J. Benson, M. A. Meyers, Void growth in metals: Atomistic calculations, Acta Mater. 56 (2008) 3874-3886.

[17] G. P. Potirniche, M. F. Horstemeyer, G. J. Wagner, P. M. Gullett, A molecular dynamics study of void growth and coalescence in single crystal nickel, Int. J. Plast. 22 (2006) 257-278.

[18] H.-J. Chang, J. Segurado, O. R. d. l. Fuente, B. M. Pabón, J. LLorca, Molecular dynamics modeling and simulation of void growth in two dimensions, Modelling Simul. Mater. Sci. Eng. 21 (2013) 075010.

[19] E. M. Bringa, S. Traiviratana, M. A. Meyers, Void initiation in fcc 
metals: Effect of loading orientation and nanocrystalline effects, Acta Mater. 58 (2010) 4458-4477.

[20] E. T. Seppälä, J. Belak, R. E. Rudd, Effect of stress triaxiality on void growth in dynamic fracture of metals: A molecular dynamics study, Phys. Rev. B 69 (2004) 134101.

[21] J. Marian, J. Knap, M. Ortiz, Nanovoid deformation in aluminum under simple shear, Acta Mater. 53 (2005) 2893-2900.

[22] M. Ponga, M. Ortiz, M. P. Ariza, Finite-temperature non-equilibrium quasi-continuum analysis of nanovoid growth in copper at low and high strain rates, Mech. Mater. 90 (2015) 253-267.

[23] S. Z. Xu, Z. M. Hao, Y. Q. Su, Y. Yu, Q. Wan, W. J. Hu, An analysis on nanovoid growth in body-centered cubic single crystalline vanadium, Comput. Mater. Sci. 50 (2011) 2411-2421.

[24] S. J. Wang, H. Wang, K. Du, W. Zhang, M. L. Sui, S. X. Mao, Deformation-induced structural transition in body-centred cubic molybdenum, Nature Comm. 5 (2014) 3433.

[25] S. Z. Xu, Z. M. Hao, Q. Wan, A molecular dynamics study of void interaction in copper, IOP Conf. Ser.: Mater. Sci. Eng. 10 (2010) 012175.

[26] T. Hatano, H. Matsui, Molecular dynamics investigation of dislocation pinning by a nanovoid in copper, Phys. Rev. B 72 (2005) 094105.

[27] V. A. Lubarda, M. S. Schneider, D. H. Kalantar, B. A. Remington, 
M. A. Meyers, Void growth by dislocation emission, Acta Mater. 52 (2004) 1397-1408.

[28] B. Chen, X. Peng, J. Fan, S. Chen, An elastoplastic constitutive description based on an ellipsoidal void model, Mater. Sci. Eng. A 423 (2006) 230-236.

[29] Y. Cui, Z. Chen, Molecular dynamics modeling on the role of initial void geometry in a thin aluminum film under uniaxial tension, Modelling Simul. Mater. Sci. Eng. 23 (2015) 085011.

[30] Y. Cui, Z. Chen, Molecular dynamics simulation of the influence of elliptical void interaction on the tensile behavior of aluminum, Comput. Mater. Sci. 108, Part A (2015) 103-113.

[31] J. Wen, Y. Huang, K. C. Hwang, C. Liu, M. Li, The modified Gurson model accounting for the void size effect, Int. J. Plast. 21 (2005) 381395.

[32] Y. Mishin, M. J. Mehl, D. A. Papaconstantopoulos, A. F. Voter, J. D. Kress, Structural stability and lattice defects in copper: Ab initio, tight-binding, and embedded-atom calculations, Phys. Rev. B 63 (2001) 224106.

[33] S. Plimpton, Fast parallel algorithms for short-range molecular dynamics, J. Comput. Phys. 117 (1995) 1-19.

[34] A. Stukowski, Visualization and analysis of atomistic simulation data with OVITO - the Open Visualization Tool, Modelling Simul. Mater. Sci. Eng. 18 (2010) 015012. 
[35] A. Stukowski, Structure identification methods for atomistic simulations of crystalline materials, Modelling Simul. Mater. Sci. Eng. 20 (2012) 045021.

[36] Y. Su, Void evolution, 2016. URL: http://www.prism.gatech. edu/ ysu65/codes/void/voids.html.

[37] J. Towns, T. Cockerill, M. Dahan, I. Foster, K. Gaither, A. Grimshaw, V. Hazlewood, S. Lathrop, D. Lifka, G. D. Peterson, R. Roskies, J. R. Scott, N. Wilkins-Diehr, XSEDE: Accelerating scientific discovery, Comput. Sci. Eng. 16 (2014) 62-74.

[38] J. Besson, Continuum models of ductile fracture: A review, Int. J. Damage Mech. 19 (2010) 3-52.

[39] J. J. Möller, E. Bitzek, On the influence of crack front curvature on the fracture behavior of nanoscale cracks, Eng. Fract. Mech. 150 (2015) 197-208. 